\title{
ABSTRACT KOROVKIN THEOREMS VIA RELATIVE MODULAR CONVERGENCE FOR DOUBLE SEQUENCES OF LINEAR OPERATORS
}

\author{
Sevda Yıldız and Kamil Demirci
}

\begin{abstract}
(C) by University of Niš, Serbia | Creative Commons Licence: CC BY-NC-ND Abstract. We will obtain an abstract version of the Korovkin type approximation theorems with respect to the concept of statistical relative convergence in modular spaces for double sequences of positive linear operators. We will give an application showing that our results are stronger than classical ones. We will also study an extension to non-positive operators.

Keywords: Korovkin type approximation; modular spaces; statistical relative convergence; non-positive operators.
\end{abstract}

\section{Introduction}

As we know, Korovkin([15]) proved an approximation theorem via simple and easy criterion to check if a sequence of positive linear operators converges uniformly to the function to be approximated. Many researchers studied some versions of this theorem in different spaces and Bardaro and Mantellini studied this theorem on modular spaces which is the natural generalization of $L_{p}(p>0)$, Orlicz, Lorentz, and Köthe spaces $([5])$ and so on $([7,8])$. In addition, general versions of the Korovkin theorem were studied in which a various kind of convergence methods is used, particularly statistical convergence methods([2, 3, 14, 22]). More recently, Demirci and Orhan ([9]) have introduced statistical relative uniform convergence of single sequences by using the notions of the natural density and the relative uniform convergence. Then, many researchers defined some versions of this interesting convergence method and proved Korovkin type approximation theorems for single and double sequences of linear operators in different spaces (see [11, 12, 13, 21, 23]). In [10], we studied Korovkin type theorems in modular function spaces for functions defined on a compact set $I^{2}$ where $I=[a, b]$, using the classical test set $\left\{1, x, y, x^{2}+y^{2}\right\}$. In this paper, we will study generalized versions of the Korovkin

Received August 08, 2018; accepted October 29, 2019

2010 Mathematics Subject Classification. Primary 46E30; Secondary 40A35, 41A36 
type approximation theorems for a double sequence of positive linear operators $\left(T_{m, n}\right)$ acting on an abstract modular function space. Then, we will give an application showing that our results are non-trivial extensions of the existing ones. Finally, we will study an extension to non-positive linear operators.

\section{Preliminaries}

Now we shall recall some well known notations and properties of modular spaces.

Assume $G$ be a locally compact Hausdorff topological space endowed with a uniform structure $\mathcal{U} \subset 2^{G \times G}$ that generates the topology of $G$ (see, [17]). Let $\mathcal{B}$ be the $\sigma$-algebra of all Borel subsets of $G$ and $\mu: \mathcal{B} \rightarrow \mathbb{R}$ is a positive $\sigma$-finite regular measure. Let $L^{0}(G)$ be the space of all real valued $\mu$-measurable functions on $G$ with identification up to sets of measure $\mu$ zero, $C(G)$ be the space of all continuous real valued functions on $G, C_{b}(G)$ be the space of all continuous real valued and bounded functions on $G$ and $C_{c}(G)$ be the subspace of $C_{b}(G)$ of all functions with compact support on $G$. In this case, we say that a functional $\rho: L^{0}(G) \rightarrow[0, \infty]$ is a modular on $L^{0}(G)$ if it satisfies the following conditions:

(i) $\rho(f)=0$ if and only if $f=0 \mu$-almost everywhere on $G$,

(ii) $\rho(-f)=\rho(f)$ for every $f \in L^{0}(G)$,

(iii) $\rho(\alpha f+\beta g) \leq \rho(f)+\rho(g)$ for every $f, g \in L^{0}(G)$ and for any $\alpha, \beta \geq 0$ with $\alpha+\beta=1$.

A modular $\rho$ is $N$-quasi convex if there exists a constant $N \geq 1$ such that the inequality

$$
\rho(\alpha f+\beta g) \leq N \alpha \rho(N f)+N \beta \rho(N g)
$$

holds for every $f, g \in L^{0}(G), \alpha, \beta \geq 0$ with $\alpha+\beta=1$. Note that if $N=1$, then $\rho$ is called convex. Furthermore, a modular $\rho$ is $N$-quasi semiconvex if there exists a constant $N \geq 1$ such that

$$
\rho(\alpha f) \leq N \alpha \rho(N f)
$$

holds for every $f \in L^{0}(G)$ and $\alpha \in(0,1]$.

The modular space $L^{\rho}(G)$ generated by $\rho$, is given by

$$
L^{\rho}(G):=\left\{f \in L^{0}(G): \lim _{\lambda \rightarrow 0^{+}} \rho(\lambda f)=0\right\}
$$

and the space of the finite elements of $L^{\rho}(G)$, is given by

$$
E^{\rho}(G):=\left\{f \in L^{\rho}(G): \rho(\lambda f)<\infty \text { for all } \lambda>0\right\} .
$$

Also, note that if $\rho$ is $N$-quasi semiconvex, then the space

$$
\left\{f \in L^{0}(G): \rho(\lambda f)<\infty \text { for some } \lambda>0\right\}
$$


coincides with $L^{\rho}(G)$.

We will need the following notions.

A modular $\rho$ is said to be monotone if $\rho(f) \leq \rho(g)$ for $|f| \leq|g|$. A modular $\rho$ is finite if $\chi_{A} \in L^{\rho}(G)$ whenever $A \in \mathcal{B}$ with $\mu(A)<\infty$. A modular $\rho$ is strongly finite if $\chi_{A} \in E^{\rho}(G)$ for all $A \in \mathcal{B}$ such that $\mu(A)<\infty$ and a modular $\rho$ is said to be absolutely continuous if there exists an $\alpha>0$ such that: for every $f \in L^{0}(G)$ with $\rho(f)<\infty$, the following conditions hold:

○ for each $\varepsilon>0$ there exists a set $A \in \mathcal{B}$ such that $\mu(A)<\infty$ and $\rho\left(\alpha f \chi_{G \backslash A}\right) \leq$ $\varepsilon$

o for every $\varepsilon>0$ there is a $\delta>0$ with $\rho\left(\alpha f \chi_{B}\right) \leq \varepsilon$ for every $B \in \mathcal{B}$ with $\mu(B)<\delta$.

If a modular $\rho$ is monotone and finite, then $C(G) \subset L^{\rho}(G)$. If $\rho$ is monotone and strongly finite, then $C(G) \subset E^{\rho}(G)$. Also, if $\rho$ is monotone, strongly finite and absolutely continuous, $\overline{C_{c}(G)}=L^{\rho}(G)$ with respect to the modular convergence (for details and properties see also $[16,18,20]$ ).

Now we recall the statistical relative modular and strong convergence for double sequences (see also [10]).

Definition 2.1. Let $\left(f_{m, n}\right)$ be a double function sequence whose terms belong to $L^{\rho}(G)$. Then, $\left(f_{m, n}\right)$ is said to be statistically relatively modularly convergent to a function $f \in L^{\rho}(G)$ if there exists a function $\sigma(u)$, called a scale function $\sigma \in L^{0}(G),|\sigma(u)| \neq 0$ such that

$$
s t_{2}-\lim _{m, n} \rho\left(\lambda_{0}\left(\frac{f_{m, n}-f}{\sigma}\right)\right)=0 \text { for some } \lambda_{0}>0 .
$$

Also, $\left(f_{m, n}\right)$ is statistically relatively $F-$ norm convergent (or, statistically relatively strongly convergent) to $f$ iff

$$
s t_{2}-\lim _{m, n} \rho\left(\lambda\left(\frac{f_{m, n}-f}{\sigma}\right)\right)=0 \text { for every } \lambda>0 .
$$

The two notions of convergence are equivalent if and only if the modular satisfies a $\Delta_{2}$-condition, i.e. there exists a constant $M>0$ such that $\rho(2 f) \leq M \rho(f)$ for every $f \in L^{0}(G)$, see [19].

Note that if the scale function is a non-zero constant, then statistical modular convergence is the special case of statistical relative modular convergence. Moreover, if $\sigma(u)$ is bounded, statistical relative modular convergence implies statistical modular convergence. However, if $\sigma(u)$ is unbounded, then statistical relative modular convergence does not imply statistical modular convergence. 


\section{Korovkin type approximation theorems}

We now prove some Korovkin type theorems with respect to an abstract finite set of test functions $e_{0}, e_{1}, \ldots, e_{k}$ in the sense of the statistical relative convergence.

Let $\mathbb{T}=\left(T_{m, n}\right)$ be a double sequence of positive linear operators from $D$ into $L^{0}(G)$ with $C_{b}(G) \subset D \subset L^{0}(G)$. Let $\rho$ be monotone and finite modular on $L^{0}(G)$ and $\sigma \in L^{0}(G)$ is an unbounded function satisfying $\sigma(u) \neq 0$. Assume further that the double sequence $\mathbb{T}$, together with modular $\rho$, satisfies the following property:

there exists a subset $X_{\mathbb{T}} \subset D \cap L^{\rho}(G)$ with $C_{b}(G) \subset X_{\mathbb{T}}$ such that the inequality

$$
s t_{2}-\limsup _{m, n} \rho\left(\lambda\left(\frac{T_{m, n} h}{\sigma}\right)\right) \leq R \rho(\lambda h)
$$

holds for every $h \in X_{\mathbb{T}}, \lambda>0$ and for an absolute positive constant $R$.

Set $e_{0}(v) \equiv 1$ for all $v \in G$, let $e_{r}, r=1,2, \ldots, k$ and $a_{r}, r=0,1,2, \ldots, k$, be functions in $C_{b}(G)$. Put

$$
P_{u}(v)=\sum_{r=0}^{k} a_{r}(u) e_{r}(v), u, v \in G
$$

and suppose that $P_{u}(v), u, v \in G$, satisfies the following properties:

(P1) $P_{u}(u)=0$, for all $u \in G$,

$(P 2)$ for every neighbourhood $U \in \mathcal{U}$ there is a positive real number $\eta$ with $P_{u}(v) \geq$ $\eta$ whenever $u, v \in G,(u, v) \notin U$ (see for examples [4]).

In order to obtain our main theorem, we will first give the following result.

Theorem 3.1. Let $\rho$ be a monotone, strongly finite and $N$-quasi semiconvex modular. Suppose that $e_{r}$ and $a_{r}, r=0,1,2, \ldots, k$, satisfy properties $(P 1)$ and $(P 2)$. Let $\mathbb{T}=\left(T_{m, n}\right)$ be a double sequence of positive linear operators from $D$ into $L^{0}(G)$ and assume that $\sigma_{r}(u)$ is an unbounded function satisfying $\left|\sigma_{r}(u)\right| \geq b_{r}>0$ $(r=0,1,2, \ldots, k)$. If

$$
s t_{2}-\lim _{m, n} \rho\left(\lambda_{0}\left(\frac{T_{m, n} e_{r}-e_{r}}{\sigma_{r}}\right)\right)=0 \text { for some } \lambda_{0}>0,
$$

$r=0,1,2, \ldots, k$, in $L^{\rho}(G)$ then for every $f \in C_{c}(G)$

$$
s t_{2}-\lim _{m, n} \rho\left(\gamma\left(\frac{T_{m, n} f-f}{\sigma}\right)\right)=0 \text { for some } \gamma>0
$$

in $L^{\rho}(G)$ where $\sigma(u)=\max \left\{\left|\sigma_{r}(u)\right|: r=0,1,2, \ldots, k\right\}$. If

$$
s t_{2}-\lim _{m, n} \rho\left(\lambda\left(\frac{T_{m, n} e_{r}-e_{r}}{\sigma_{r}}\right)\right)=0 \text { for every } \lambda>0,
$$


$r=0,1,2, \ldots, k$, in $L^{\rho}(G)$ then for every $f \in C_{c}(G)$

$$
s t_{2}-\lim _{m, n} \rho\left(\lambda\left(\frac{T_{m, n} f-f}{\sigma}\right)\right)=0 \text { for every } \lambda>0
$$

in $L^{\rho}(G)$ where $\sigma(u)=\max \left\{\left|\sigma_{r}(u)\right|: r=0,1,2, \ldots, k\right\}$.

Proof. We first claim that, for every $f \in C_{c}(G)$,

$$
s t_{2}-\lim _{m, n} \rho\left(\gamma\left(\frac{T_{m, n} f-f}{\sigma}\right)\right)=0 \text { for some } \gamma>0 .
$$

To see this assume that $f \in C_{c}(G)$. Then, since $G$ is endowed with the uniform structure $\mathcal{U}, f$ is uniformly continuous and bounded on $G$. By the uniform continuity of $f$, choose $\varepsilon \in(0,1]$, there exists a set $U \in \mathcal{U}$ such that $|f(u)-f(v)| \leq \varepsilon$ whenever $u, v \in G,(u, v) \in U$.

For all $u, v \in G$ let $P_{u}(v)$ be as in (3.2), and $\eta>0$ satisfy condition (P2). Then for $u, v \in G,(u, v) \notin U$, we have $|f(u)-f(v)| \leq \frac{2 M}{\eta} P_{u}(v)$ where $M:=\sup _{v \in G}|f(v)|$. Therefore, in any case we get $|f(u)-f(v)| \leq \varepsilon+\frac{2 M}{\eta} P_{u}(v)$ for all $u, v \in G$, namely,

$$
-\varepsilon-\frac{2 M}{\eta} P_{u}(v) \leq f(u)-f(v) \leq \varepsilon+\frac{2 M}{\eta} P_{u}(v) .
$$

Since $T_{m, n}$ is linear and positive, by applying $T_{m, n}$ to (3.6) for every $m, n \in \mathbb{N}$ we have

$$
\begin{aligned}
-\varepsilon T_{m, n}\left(e_{0} ; u\right)-\frac{2 M}{\eta} T_{m, n}\left(P_{u} ; u\right) & \leq f(u) T_{m, n}\left(e_{0} ; u\right)-T_{m, n}(f ; u) \\
& \leq \varepsilon T_{m, n}\left(e_{0} ; u\right)+\frac{2 M}{\eta} T_{m, n}\left(P_{u} ; u\right) .
\end{aligned}
$$

Hence

$$
\begin{aligned}
\left|T_{m, n}(f ; u)-f(u)\right| \leq \quad & \left|T_{m, n}(f ; u)-f(u) T_{m, n}\left(e_{0} ; u\right)\right| \\
& +|f(u)|\left|T_{m, n}\left(e_{0} ; u\right)-e_{0}(u)\right| \\
\leq \quad & \varepsilon T_{m, n}\left(e_{0} ; u\right)+\frac{2 M}{\eta} T_{m, n}\left(P_{u} ; u\right) \\
& +M\left|T_{m, n}\left(e_{0} ; u\right)-e_{0}(u)\right| \\
\leq \quad & +(\varepsilon+M)\left|T_{m, n}\left(e_{0} ; u\right)-e_{0}(u)\right| \\
& +\frac{2 M}{\eta} \sum_{r=0}^{k} a_{r}(u)\left|T_{m, n}\left(e_{r} ; u\right)-e_{r}(u)\right| .
\end{aligned}
$$

Let $\gamma>0$. Now for each $r=0,1,2, \ldots, k$ and $u \in G$, choose $M_{0}>0$ such that $\left|a_{r}(u)\right| \leq M_{0}$ and multiplying the both sides of the above inequality by $\frac{1}{|\sigma(u)|}$, the last inequality gives that

$$
\gamma\left|\frac{T_{m, n}(f ; u)-f(u)}{\sigma(u)}\right| \leq \frac{\gamma \varepsilon}{|\sigma(u)|}+K \gamma \sum_{r=0}^{k}\left|\frac{T_{m, n}\left(e_{r} ; u\right)-e_{r}(u)}{\sigma(u)}\right|
$$


where $K:=\varepsilon+M+\frac{2 M}{\eta} M_{0}$. Now, applying the modular $\rho$ to both sides of the above inequality, since $\rho$ is monotone and $\sigma(u)=\max \left\{\left|\sigma_{r}(u)\right|: r=0,1,2, \ldots, k\right\}$, we get

$$
\rho\left(\gamma\left(\frac{T_{m, n} f-f}{\sigma}\right)\right) \leq \rho\left(\frac{\gamma \varepsilon}{|\sigma|}+K \gamma \sum_{r=0}^{k}\left|\frac{T_{m, n} e_{r}-e_{r}}{\sigma_{r}}\right|\right) .
$$

Thus, we can see that

$$
\rho\left(\gamma\left(\frac{T_{m, n} f-f}{\sigma}\right)\right) \leq \rho\left(\frac{(k+2) \gamma \varepsilon}{\sigma}\right)+\sum_{r=0}^{k} \rho\left((k+2) K \gamma\left(\frac{T_{m, n} e_{r}-e_{r}}{\sigma_{r}}\right)\right) .
$$

Let $\lambda_{0}>0$ be as in the hypothesis (3.3), such $\lambda_{0}>0$, by hypothesis, does exist. Let $\gamma>0$ be with $(k+2) K \gamma \leq \lambda_{0}$ and since $\rho$ is $N$-quasi semiconvex and strongly finite, we have,

$$
\rho\left(\gamma\left(\frac{T_{m, n} f-f}{\sigma}\right)\right) \leq N \varepsilon \rho\left(\frac{(k+2) \gamma N}{\sigma}\right)+\sum_{r=0}^{k} \rho\left(\lambda_{0}\left(\frac{T_{m, n} e_{r}-e_{r}}{\sigma_{r}}\right)\right) .
$$

For a given $\varepsilon^{*}>0$, choose an $\varepsilon \in(0,1]$ such that $N \varepsilon \rho\left(\frac{(k+2) \gamma N}{\sigma}\right)<\varepsilon^{*}$. Now define the following sets:

$$
\begin{aligned}
S_{\gamma}: & \left.=\left\{(m, n): \rho\left(\gamma\left(\frac{T_{m, n} f-f}{\sigma}\right)\right)\right) \geq \varepsilon^{*}\right\} \\
S_{\gamma, r}: & =\left\{(m, n): \rho\left(\lambda_{0}\left(\frac{T_{m, n} e_{r}-e_{r}}{\sigma_{r}}\right)\right) \geq \frac{\varepsilon^{*}-N \varepsilon \rho\left(\frac{(k+2) \gamma N}{\sigma}\right)}{k+1}\right\},
\end{aligned}
$$

where $r=0,1,2, \ldots, k$. Then, it is easy to see that $S_{\gamma} \subseteq \bigcup_{r=0}^{k} S_{\gamma, r}$. So, we can see that

$$
\delta_{2}\left(S_{\gamma}\right) \leq \sum_{r=0}^{k} \delta_{2}\left(S_{\gamma, r}\right)
$$

Using the hypothesis (3.3), we get

$$
\delta_{2}\left(S_{\gamma}\right)=0,
$$

which proves our claim (3.5).

The last part of theorem can be proved similarly to the first one.

Now, we can give our main theorem of this paper.

Theorem 3.2. Let $\rho$ be a monotone, strongly finite, absolutely continuous and $N$-quasi semiconvex modular. Suppose that $e_{r}$ and $a_{r}, r=0,1,2, \ldots, k$, satisfy 
properties $(P 1)$ and $(P 2)$. Let $\mathbb{T}=\left(T_{m, n}\right)$ be a double sequence of positive linear operators satisfying (3.1) and assume that $\sigma_{r}(u)$ is an unbounded function satisfying $\left|\sigma_{r}(u)\right| \geq b_{r}>0(r=0,1,2, \ldots, k)$. If

$$
s t_{2}-\lim _{m, n} \rho\left(\lambda\left(\frac{T_{m, n} e_{r}-e_{r}}{\sigma_{r}}\right)\right)=0 \text { for every } \lambda>0,
$$

$r=0,1,2, \ldots, k$ in $L^{\rho}(G)$, then for every $f \in D \cap L^{\rho}(G)$ with $f-C_{b}(G) \subset X_{\mathbb{T}}$,

$$
s t_{2}-\lim _{m, n} \rho\left(\lambda_{0}\left(\frac{T_{m, n} f-f}{\sigma}\right)\right)=0 \text { for some } \lambda_{0}>0
$$

in $L^{\rho}(G)$ where $\sigma(u)=\max \left\{\left|\sigma_{r}(u)\right|: r=0,1,2, \ldots, k\right\}$ and $D, X_{\mathbb{T}}$ are as before.

Proof. Let $f \in D \cap L^{\rho}(G)$ with $f-C_{b}(G) \subset X_{\mathbb{T}}$. It is known from $[6,18]$ that there exists a sequence $\left(g_{k, j}\right) \subset C_{c}(G)$ such that $\rho\left(3 \lambda_{0}^{*} f\right)<\infty$ and $P-$ $\lim _{k, j} \rho\left(3 \lambda_{0}^{*}\left(g_{k, j}-f\right)\right)=0$ for some $\lambda_{0}^{*}>0$. This means that, for every $\varepsilon>0$, there is a positive number $k_{0}=k_{0}(\varepsilon)$ with

$$
\rho\left(3 \lambda_{0}^{*}\left(g_{k, j}-f\right)\right)<\varepsilon \text { for every } k, j \geq k_{0} .
$$

For all $m, n \in \mathbb{N}$, by linearity and positivity of the operators $T_{m, n}$, we have

$$
\begin{aligned}
\lambda_{0}^{*}\left|T_{m, n}(f ; u)-f(u)\right| \leq & \lambda_{0}^{*}\left|T_{m, n}\left(f-g_{k_{0}, k_{0}} ; u\right)\right|+\lambda_{0}^{*}\left|T_{m, n}\left(g_{k_{0}, k_{0}} ; u\right)-g_{k_{0}, k_{0}}(u)\right| \\
& +\lambda_{0}^{*}\left|g_{k_{0}, k_{0}}(u)-f(u)\right|
\end{aligned}
$$

holds for every $u \in G$. Now, applying the modular $\rho$ in the last inequality and using the monotonicity of $\rho$ and moreover multiplying both sides of the above inequality by $\frac{1}{|\sigma(u)|}$, we get

$$
\begin{aligned}
& \rho\left(\lambda_{0}^{*}\left(\frac{T_{m, n} f-f}{\sigma}\right)\right) \\
\leq & \rho\left(3 \lambda_{0}^{*}\left(\frac{T_{m, n}\left(f-g_{k_{0}, k_{0}}\right)}{\sigma}\right)\right)+\rho\left(3 \lambda_{0}^{*}\left(\frac{T_{m, n} g_{k_{0}, k_{0}}-g_{k_{0}, k_{0}}}{\sigma}\right)\right) \\
& +\rho\left(3 \lambda_{0}^{*}\left(\frac{g_{k_{0}, k_{0}}-f}{\sigma}\right)\right) .
\end{aligned}
$$

Hence, observing $|\sigma| \geq b>0,\left(b=\max \left\{b_{r}: r=0,1,2, \ldots, k\right\}\right)$, we can write that

$$
\begin{aligned}
& \rho\left(\lambda_{0}^{*}\left(\frac{T_{m, n} f-f}{\sigma}\right)\right) \\
\leq & \rho\left(3 \lambda_{0}^{*}\left(\frac{T_{m, n}\left(f-g_{k_{0}, k_{0}}\right)}{\sigma}\right)\right)+\rho\left(3 \lambda_{0}^{*}\left(\frac{T_{m, n} g_{k_{0}, k_{0}}-g_{k_{0}, k_{0}}}{\sigma}\right)\right) \\
& +\rho\left(\frac{3 \lambda_{0}^{*}}{b}\left(g_{k_{0}, k_{0}}-f\right)\right) .
\end{aligned}
$$


Then using the (3.8) in (3.9), we have

$$
\begin{aligned}
\rho\left(\lambda_{0}^{*}\left(\frac{T_{m, n} f-f}{\sigma}\right)\right) \leq & \varepsilon+\rho\left(3 \lambda_{0}^{*}\left(\frac{T_{m, n}\left(f-g_{k_{0}, k_{0}}\right)}{\sigma}\right)\right) \\
& +\rho\left(3 \lambda_{0}^{*}\left(\frac{T_{m, n} g_{k_{0}, k_{0}}-g_{k_{0}, k_{0}}}{\sigma}\right)\right) .
\end{aligned}
$$

By property (3.1) and also using the facts that $g_{k_{0}, k_{0}} \in C_{c}(G)$ and $f-g_{k_{0}, k_{0}} \in X_{\mathbb{T}}$, we obtain

$$
\begin{aligned}
& s t_{2}-\limsup _{m, n} \rho\left(\lambda_{0}^{*}\left(\frac{T_{m, n} f-f}{\sigma}\right)\right) \\
\leq & \varepsilon+R \rho\left(3 \lambda_{0}^{*}\left(f-g_{k_{0}, k_{0}}\right)\right)+s t_{2}-\limsup _{m, n} \rho\left(3 \lambda_{0}^{*}\left(\frac{T_{m, n} g_{k_{0}, k_{0}}-g_{k_{0}, k_{0}}}{\sigma}\right)\right) \\
\leq & \varepsilon(1+R)+s t_{2}-\limsup _{m, n} \rho\left(3 \lambda_{0}^{*}\left(\frac{T_{m, n} g_{k_{0}, k_{0}}-g_{k_{0}, k_{0}}}{\sigma}\right)\right)
\end{aligned}
$$

also, resulting from previous theorem,

$$
\begin{aligned}
0 & =s t_{2}-\lim _{m, n} \rho\left(3 \lambda_{0}^{*}\left(\frac{T_{m, n} g_{k_{0}, k_{0}}-g_{k_{0}, k_{0}}}{\sigma}\right)\right) \\
& =s t_{2}-\limsup _{m, n} \rho\left(3 \lambda_{0}^{*}\left(\frac{T_{m, n} g_{k_{0}, k_{0}}-g_{k_{0}, k_{0}}}{\sigma}\right)\right)
\end{aligned}
$$

which gives

$$
0 \leq s t_{2}-\limsup _{m, n} \rho\left(\lambda_{0}^{*}\left(\frac{T_{m, n} f-f}{\sigma}\right)\right) \leq \varepsilon(1+R) .
$$

From arbitrariety of $\varepsilon>0$, it follows that

$$
s t_{2}-\limsup _{m, n} \rho\left(\lambda_{0}^{*}\left(\frac{T_{m, n} f-f}{\sigma}\right)\right)=0 .
$$

Furthermore,

$$
s t_{2}-\lim _{m, n} \rho\left(\lambda_{0}^{*}\left(\frac{T_{m, n} f-f}{\sigma}\right)\right)=0,
$$

and this completes the proof.

Remark 3.1. Note that, in Theorem 3.2, in general it is not possible to obtain statistical relative strong convergence unless the modular $\rho$ satisfies the $\Delta_{2}$-condition.

If one replaces the scale function by a nonzero constant, then the condition (3.1) is reduced to

$$
s t_{2}-\limsup _{m, n} \rho\left(\lambda\left(T_{m, n} h\right)\right) \leq R \rho(\lambda h)
$$

for every $h \in X_{\mathbb{T}}, \lambda>0$ and for an absolute positive constant $R$. In this case, the next result immediately follows from our Theorem 3.2. 
Corollary 3.1. Let $\rho$ be a monotone, strongly finite, absolutely continuous and $N$-quasi semiconvex modular. Suppose that $e_{r}$ and $a_{r}, r=0,1,2, \ldots, k$, satisfy properties $(P 1)$ and $(P 2)$. Let $\mathbb{T}=\left(T_{m, n}\right)$ be a double sequence of positive linear operators satisfying (3.10). If $\left(T_{m, n} e_{r}\right)$ is statistically strongly convergent to $e_{r}$, $r=0,1,2, \ldots, k$, in $L^{\rho}(G)$, then $\left(T_{m, n} f\right)$ is statistically modularly convergent to $f$ in $L^{\rho}(G)$ such that $f$ is any function belonging to $D \cap L^{\rho}(G)$ with $f-C_{b}(G) \subset X_{\mathbb{T}}$.

If one replaces the statistical limit by the Pringsheim limit, then the condition (3.1) is reduced to

$$
P-\limsup _{m, n} \rho\left(\lambda\left(\frac{T_{m, n} h}{\sigma}\right)\right) \leq R \rho(\lambda h)
$$

for every $h \in X_{\mathbb{T}}, \lambda>0$ and for an absolute positive constant $R$. In this case, the following result immediately follows from our Theorem 3.2.

Corollary 3.2. Let $\rho$ be a monotone, strongly finite, absolutely continuous and $N$-quasi semiconvex modular. Suppose that $e_{r}$ and $a_{r}, r=0,1,2, \ldots, k$, satisfy properties $(P 1)$ and $(P 2)$. Let $\mathbb{T}=\left(T_{m, n}\right)$ be a double sequence of positive linear operators satisfying (3.11) and assume that $\sigma_{r}(u)$ is an unbounded function satisfying $\left|\sigma_{r}(u)\right| \geq b_{r}>0(r=0,1,2, \ldots, k)$. If $\left(T_{m, n} e_{r}\right)$ is relatively strongly convergent to $e_{r}$ to the scale function $\sigma_{r}, r=0,1,2, \ldots, k$, in $L^{\rho}(G)$ then $\left(T_{m, n} f\right)$ is relatively modularly convergent to $f$ to the scale function $\sigma$ in $L^{\rho}(G)$ where $\sigma(u)=$ $\max \left\{\left|\sigma_{r}(u)\right|: r=0,1,2, \ldots, k\right\}$ and $f$ is any function belonging to $D \cap L^{\rho}(G)$ with $f-C_{b}(G) \subset X_{\mathbb{T}}$.

Now, we give an application showing that in general, our results are stronger than classical ones.

Example 3.1. Let us consider $G=[0,1]^{2} \subset \mathbb{R}^{2}$ and let $\varphi:[0, \infty) \rightarrow[0, \infty)$ be a continuous and convex function with $\varphi(0)=0, \varphi(x)>0$ for any $x>0$ and $\lim _{x \rightarrow \infty} \varphi(x)=\infty$. Then, the functional $\rho^{\varphi}$ defined by

$$
\rho^{\varphi}(f):=\int_{0}^{1} \int_{0}^{1} \varphi(|f(x, y)|) d x d y \quad \text { for } f \in L^{0}(G),
$$

is a convex modular on $L^{0}(G)$ and

$$
L^{\varphi}(G):=\left\{f \in L^{0}(G): \rho^{\varphi}(\lambda f)<+\infty \quad \text { for some } \lambda>0\right\}
$$

is the Orlics space generated by $\varphi$.

For every $(x, y) \in G$, let $e_{0}(x, y)=a_{3}(x, y)=1, e_{1}(x, y)=x, e_{2}(x, y)=y, e_{3}(x, y)=$ $a_{0}(x, y)=x^{2}+y^{2}, a_{1}(x, y)=-2 x, a_{2}(x, y)=-2 y$. For every $m, n \in \mathbb{N}, u_{1}, u_{2} \in[0,1]$, let $K_{m, n}\left(u_{1}, u_{2}\right)=(m+1)(n+1) u_{1}^{m} u_{2}^{n}$ and for $f \in C(G)$ and $x, y \in[0,1]$ set

$$
M_{m, n}(f ; x, y)=\int_{0}^{1} \int_{0}^{1} K_{m, n}\left(u_{1}, u_{2}\right) f\left(u_{1} x, u_{2} y\right) d u_{1} d u_{2} .
$$


Then we get

$$
\begin{aligned}
& \int_{0}^{1} \int_{0}^{1} K_{m, n}\left(u_{1}, u_{2}\right) d u_{1} d u_{2} \\
= & (m+1)\left(\int_{0}^{1} u_{1}^{m} d u_{1}\right)(n+1)\left(\int_{0}^{1} u_{2}^{n} d u_{2}\right)=1,
\end{aligned}
$$

and hence, $M_{m, n}\left(e_{0} ; x, y\right)=e_{0}(x, y)=1$. Also, we know from [3] that

$$
\begin{aligned}
& \left|M_{m, n}\left(e_{1} ; x, y\right)-e_{1}(x, y)\right| \leq \frac{1}{m+2},\left|M_{m, n}\left(e_{2} ; x, y\right)-e_{2}(x, y)\right| \leq \frac{1}{n+2}, \\
& \left|M_{m, n}\left(e_{1}^{2} ; x, y\right)-e_{1}^{2}(x, y)\right| \leq \frac{2}{m+3},\left|M_{m, n}\left(e_{2}^{2} ; x, y\right)-e_{2}^{2}(x, y)\right| \leq \frac{2}{n+3},
\end{aligned}
$$

and for each $m, n \geq 2, f \in L^{\varphi}(G)$ we get $\rho^{\varphi}\left(M_{m, n} f\right) \leq 32 \rho^{\varphi}(f)$. Moreover, $\left(M_{m, n}\right)$ satisfies the condition (14) in [22] with $X_{\mathbb{M}}=L^{\varphi}(G)$ and $\left(M_{m, n} f\right)$ is modulary convergent to $f \in L^{\varphi}(G)$. Using the operators $\mathbb{M}=\left(M_{m, n}\right)$, we define the double sequence of positive linear operators $\mathbb{T}=\left(T_{m, n}\right)$ on $L^{\varphi}(G)$ as follows:

$$
T_{m, n}(f ; x, y)=\left(1+g_{m, n}(x, y)\right) M_{m, n}(f ; x, y), \text { for } f \in L^{\varphi}(G),
$$

$x, y \in[0,1]$ and $m, n \in \mathbb{N}$, where $g_{m, n}: G \rightarrow \mathbb{R}$ defined by

$$
g_{m, n}(x, y)= \begin{cases}1, & m=k^{2} \text { and } n=l^{2} \\ \left(m^{2}+1\right) n^{3} x y, & (x, y) \in\left(0, \frac{1}{m}\right) \times\left(0, \frac{1}{n}\right) ; m \neq k^{2} \text { and } n \neq l^{2} \\ 0, & (x, y) \notin\left(0, \frac{1}{m}\right) \times\left(0, \frac{1}{n}\right) ; m \neq k^{2} \text { and } n \neq l^{2}\end{cases}
$$

$k, l=1,2, \ldots$ If $\varphi(x)=x^{p}$ for $1 \leq p<\infty, x \geq 0$ then $L^{\varphi}(G)=L_{p}(G)$ and we have for any function $f \in L^{\varphi}(G), \rho^{\varphi}(f)=\|f\|_{p}^{p}$. From now on we have $p=1$.

It is clear that

$$
\begin{aligned}
\rho\left(\lambda_{0}\left(g_{m, n}-g\right)\right) & =\left\|\lambda_{0}\left(g_{m, n}-g\right)\right\|_{1} \\
& =\lambda_{0}\left\{\begin{array}{cc}
1, & m=k^{2} \text { and } n=l^{2} \\
\frac{\left(m^{2}+1\right) n}{4 m^{2}}, & m \neq k^{2} \text { and } n \neq l^{2}
\end{array}, k, l=1,2, \ldots,\right.
\end{aligned}
$$

where $g=0$, then $\left(g_{m, n}\right)$ does not converge statistically modularly to $g=0$. Now, we choose $\sigma_{r}(x, y)=\sigma(x, y)(r=0,1,2,3)$ where $\sigma(x, y)=\left\{\begin{array}{cc}\frac{1}{x^{2} y}, & (x, y) \in(0,1] \times(0,1] \\ 1, & \text { otherwise }\end{array}\right.$ on $L_{1}(G)$. Then, it can be seen that, for every $h \in L_{1}(G), \lambda>0$ and for positive constant $R_{0}$ that

Now, observe that

$$
s t_{2}-\limsup _{m, n}\left\|\lambda\left(\frac{T_{m, n} h}{\sigma}\right)\right\|_{1} \leq R_{0}\|\lambda h\|_{1} .
$$

$$
\begin{aligned}
& T_{m, n}\left(e_{0} ; x, y\right)-e_{0}(x, y)=g_{m, n}(x, y) \\
& T_{m, n}\left(e_{1} ; x, y\right)-e_{1}(x, y) \leq \frac{1+g_{m, n}(x, y)}{m+2}+g_{m, n}(x, y), \\
& T_{m, n}\left(e_{2} ; x, y\right)-e_{2}(x, y) \leq \frac{1+g_{m, n}(x, y)}{n+2}+g_{m, n}(x, y), \\
& T_{m, n}\left(e_{3} ; x, y\right)-e_{3}(x, y) \leq\left(1+g_{m, n}(x, y)\right)\left(\frac{2}{m+3}+\frac{2}{n+3}\right)+2 g_{m, n}(x, y) .
\end{aligned}
$$


Hence, we can see, for any $\lambda>0$, that

$$
\begin{aligned}
& \left\|\lambda\left(\frac{T_{m, n} e_{0}-e_{0}}{\sigma}\right)\right\|_{1} \\
= & \left\|\lambda\left(\frac{g_{m, n}}{\sigma}\right)\right\|_{1}=\lambda\left\{\begin{array}{cc}
1, & m=k^{2} \text { and } n=l^{2} \\
\frac{m^{2}+1}{12 m^{4}}, & m \neq k^{2} \text { and } n \neq l^{2}
\end{array}, k, l=1,2, \ldots,\right.
\end{aligned}
$$

then, we get

$$
s t_{2}-\lim _{m, n}\left\|\lambda\left(\frac{T_{m, n} e_{0}-e_{0}}{\sigma}\right)\right\|_{1}=0 .
$$

Also, we have

$$
\begin{aligned}
\left\|\lambda\left(\frac{T_{m, n} e_{1}-e_{1}}{\sigma}\right)\right\|_{1} & \leq\left\|\frac{\lambda}{\sigma}\left(\frac{1+g_{m, n}}{m+2}+g_{m, n}\right)\right\|_{1} \\
& \leq \frac{\lambda}{m+2}\left\|\frac{1+g_{m, n}}{\sigma}\right\|_{1}+\left\|\lambda\left(\frac{g_{m, n}}{\sigma}\right)\right\|_{1} \\
& \leq \frac{1}{m+2}\left(\frac{\lambda}{6}+\left\|\lambda\left(\frac{g_{m, n}}{\sigma}\right)\right\|_{1}\right)+\left\|\lambda\left(\frac{g_{m, n}}{\sigma}\right)\right\|_{1},
\end{aligned}
$$

from above inequality, since the equality (3.12), we have

$$
s t_{2}-\lim _{m, n}\left\|\lambda\left(\frac{T_{m, n} e_{1}-e_{1}}{\sigma}\right)\right\|_{1}=0 .
$$

Similarly, we get

$$
s t_{2}-\lim _{m, n}\left\|\lambda\left(\frac{T_{m, n} e_{2}-e_{2}}{\sigma}\right)\right\|_{1}=0 .
$$

Finally, since

$$
\begin{aligned}
& \left\|\lambda\left(\frac{T_{m, n} e_{3}-e_{3}}{\sigma}\right)\right\|_{1} \\
\leq & \left\|\frac{\lambda}{\sigma}\left(\left(1+g_{m, n}(x, y)\right)\left(\frac{2}{m+3}+\frac{2}{n+3}\right)+2 g_{m, n}(x, y)\right)\right\|_{1} \\
\leq & \left(\frac{2}{m+3}+\frac{2}{n+3}\right)\left(\frac{\lambda}{6}+\left\|\lambda\left(\frac{g_{m, n}}{\sigma}\right)\right\|_{1}\right)+2\left\|\lambda\left(\frac{g_{m, n}}{\sigma}\right)\right\|_{1},
\end{aligned}
$$

then,

$$
s t_{2}-\lim _{m, n}\left\|\lambda\left(\frac{T_{m, n} e_{3}-e_{3}}{\sigma}\right)\right\|_{1}=0 .
$$

So, our new operator $\mathbb{T}=\left(T_{m, n}\right)$ satisfies all conditions of Theorem 3.2 and therefore we obtain

$$
s t_{2}-\lim _{m, n}\left\|\lambda_{0}\left(\frac{T_{m, n} f-f}{\sigma}\right)\right\|_{1}=0
$$

for some $\lambda_{0}>0$, for any $f \in L_{1}(G)$. However, $\left(T_{m, n} e_{0}\right)$ is neither relatively modularly convergent to the scale function $\sigma$ nor statistically modularly convergent. Thus $\left(T_{m, n}\right)$ does not fulfil the Corollary 3.1 and 3.2. 


\section{An Extension to Non-Positive Operators}

In this section, we relax the positivity condition of linear operators in the Korovkin theorems. In $[1,3,4]$ there are some positive answers. Following this approach, we give some positive answers for statistical relative modular convergence and prove a Korovkin type approximation theorem.

Let $I$ be a bounded interval of $\mathbb{R}, C^{2}(I)$ (resp. $C_{b}^{2}(I)$ ) be the space of all functions defined on $I$, (resp. bounded and) continuous together with their first and second derivatives, $C_{+}:=\left\{f \in C_{b}^{2}(I): f \geq 0\right\}, C_{+}^{2}:=\left\{f \in C_{b}^{2}(I): f^{\prime \prime} \geq 0\right\}$.

Let $e_{r}, r=1,2, \ldots, k$ and $a_{r}, r=0,1,2, \ldots, k$, be functions in $C_{b}^{2}(I), P_{u}(v)$, $u, v \in I$, be as in (3.2), and suppose that $P_{u}(v)$ satisfies the properties $(P 1),(P 2)$ and

(P3) there is a positive real constant $S_{0}$ such that $P_{u}^{\prime \prime}(v) \geq S_{0}$ for all $u, v \in I$ (Here the second derivative is intended with respect to $v$ ).

Now we prove the following Korovkin type approximation theorem for not necessarily positive linear operators.

Theorem 4.1. Let $\rho$ and $\sigma_{r}$ be as in Theorem 3.1 and $e_{r}, a_{r}, r=0,1,2, \ldots, k$ and $P_{u}(v), u, v \in I$, satisfies the properties $(P 1),(P 2)$ and $(P 3)$. Assume that $\mathbb{T}=\left(T_{m, n}\right)$ be a double sequence of linear operators and $T_{m, n}\left(C_{+} \cap C_{+}^{2}\right) \subset C_{+}$ for all $m, n \in \mathbb{N}$. If $T_{m, n} e_{r}$ is statistically relatively modularly convergent to $e_{r}$ to the scale function $\sigma_{r}$ in $L^{\rho}(I)$ for each $r=0,1,2, \ldots, k$, then $T_{m, n} f$ is statistically relatively modularly convergent to $f$ to the scale function $\sigma$ in $L^{\rho}(I)$ for every $f \in C_{b}^{2}(I)$ where $\sigma(u)=\max \left\{\left|\sigma_{r}(u)\right|: r=0,1,2, \ldots, k\right\}$.

If $T_{m, n} e_{r}$ is statistically relatively strongly convergent to $e_{r}$ to the scale function $\sigma_{r}, r=0,1,2, \ldots, k$, in $L^{\rho}(I)$ then $T_{m, n} f$ is statistically relatively strongly convergent to $f$ to the scale function $\sigma$ in $L^{\rho}(I)$ for every $f \in C_{b}^{2}(I)$ where $\sigma(u)=\max \left\{\left|\sigma_{r}(u)\right|: r=0,1,2, \ldots, k\right\}$.

Furthermore, if $\rho$ is absolutely continuous, $\mathbb{T}$ satisfies the property (3.1) and $T_{m, n} e_{r}$ is statistically relatively strongly convergent to $e_{r}$ to the scale function $\sigma_{r}$, $r=0,1,2, \ldots, k$, in $L^{\rho}(I)$ then $T_{m, n} f$ is statistically relatively modularly convergent to $f$ to the scale function $\sigma$ in $L^{\rho}(I)$ for every $f \in D \cap L^{\rho}(G)$ with $f-C_{b}(I) \subset X_{\mathbb{T}}$ where $\sigma(u)=\max \left\{\left|\sigma_{r}(u)\right|: r=0,1,2, \ldots, k\right\}$.

Proof. Let $f \in C_{b}^{2}(I)$. Since $f$ is uniformly continuous and bounded on I, given $\varepsilon>0$ with $0<\varepsilon \leq 1$, there exists a $\delta>0$ such that $|f(u)-f(v)| \leq \varepsilon$ for all $u, v \in I,|u-v| \leq \delta$. Let $P_{u}(v), u, v \in I$, be as in (3.2) and let $\eta>0$ be associated with $\delta$, satisfying (P2). As in Theorem 3.1, for every $\beta \geq 1$ and $u, v \in I$, we have

$$
-\varepsilon-\frac{2 M \beta}{\eta} P_{u}(v) \leq f(u)-f(v) \leq \varepsilon+\frac{2 M \beta}{\eta} P_{u}(v)
$$

where $M=\sup _{v \in I}|f(v)|$. From (4.1) it follows that

$$
h_{1, \beta}(v):=\varepsilon+\frac{2 M \beta}{\eta} P_{u}(v)+f(v)-f(u) \geq 0,
$$




$$
h_{2, \beta}(v):=\varepsilon+\frac{2 M \beta}{\eta} P_{u}(v)-f(v)+f(u) \geq 0 .
$$

Let $H_{0}$ satisfy $(P 3)$. For each $v \in I$, we get

$$
h_{1, \beta}^{\prime \prime}(v) \geq \frac{2 M \beta H_{0}}{\eta}+f^{\prime \prime}(v), h_{2, \beta}^{\prime \prime}(v) \geq \frac{2 M \beta H_{0}}{\eta}-f^{\prime \prime}(v) .
$$

Because of $f^{\prime \prime}$ is bounded on $I$, we can choose $\beta \geq 1$ in such a way that $h_{1, \beta}^{\prime \prime}(v) \geq 0$, $h_{2, \beta}^{\prime \prime}(v) \geq 0$ for each $v \in I$. Hence $h_{1, \beta}, h_{2, \beta} \in C_{+} \cap C_{+}^{2}$ and then, by hypothesis

$$
T_{m, n}\left(h_{j, \beta} ; u\right) \geq 0 \text { for all } m, n \in \mathbb{N}, u \in I \text { and } j=1,2 .
$$

From (4.2)-(4.4) and the linearity of $T_{m, n}$, we get

$$
\begin{aligned}
& \varepsilon T_{m, n}\left(e_{0} ; u\right)+\frac{2 M \beta}{\eta} T_{m, n}\left(P_{u} ; u\right)+T_{m, n}(f ; u)-f(u) T_{m, n}\left(e_{0} ; u\right) \geq 0 \\
& \varepsilon T_{m, n}\left(e_{0} ; u\right)+\frac{2 M \beta}{\eta} T_{m, n}\left(P_{u} ; u\right)-T_{m, n}(f ; u)+f(u) T_{m, n}\left(e_{0} ; u\right) \geq 0
\end{aligned}
$$

thus,

$$
\begin{aligned}
-\varepsilon T_{m, n}\left(e_{0} ; u\right)-\frac{2 M \beta}{\eta} T_{m, n}\left(P_{u} ; u\right) & \leq f(u) T_{m, n}\left(e_{0} ; u\right)-T_{m, n}(f ; u) \\
& \leq \varepsilon T_{m, n}\left(e_{0} ; u\right)+\frac{2 M \beta}{\eta} T_{m, n}\left(P_{u} ; u\right) .
\end{aligned}
$$

By arguing similarly as in the proof of Theorem 3.1, multiplying the inequality by $\frac{1}{|\sigma(u)|}$, using the modular $\rho$ and for $m, n \in \mathbb{N}$, we have the assertion of the first part.

The other parts can be proved similarly as in the proofs of Theorems 3.1 and 3.2.

\section{REF EREN C ES}

1. G. A. Anastassiou and O. Duman: Towads intelligent modeling: Statistical approximation theory. Intelligent System Reference Library 14, Springer-Verlag, Berlin, Heidelberg, New York, 2011.

2. C. Bardaro, A. Boccuto, K. Demirci, I. Mantellini, S. Orhan: Triangular A-statistical approximation by double sequences of positive linear operators. Results Math. 68 (2015), 271-291.

3. C. Bardaro, A. Boccuto, K. Demirci, I. Mantellini, S. Orhan: Korovkin-type theorems for modular $\Psi-A$-statistical convergence. J. Funct. Spaces Article ID 160401, 2015 (2015), p. 11.

4. C. Bardaro, A. Boccuto, X. Dimitriou and I. Mantellini: Abstract Korovkin type theorems in modular spaces and applications. Cent. Eur. J. Math. 11(10) (2013), 1774-1784. 
5. C. BARdARo and I. MANTELlini: Korovkin's theorem in modular spaces. Commentationes Math. 47 (2007), 239-253.

6. C. BArdaro, J. Musielak, G. Vinti: Nonlinear integral operators and applications. de Gruyter Series in Nonlinear Analysis and Appl. Vol., 9 Walter de Gruyter Publ., Berlin, 2003.

7. C. BARdaro and I. Mantellini: A Korovkin Theorem in multivariate modular function spaces. J. Funct. Spaces Appl. 7 (2009), 105-120.

8. A. Boccuto and X. Dimitriou: Korovkin-type theorems for abstract modular convergence. Results in Mathematics 69 (2016), 477-495.

9. K. Demirci, S. Orhan: Statistically Relatively Uniform Convergence of Positive Linear Operators. Results in Mathematics 69 (2016), 359-367.

10. K. Demirci, S. Orhan: Statistical relative approximation on modular spaces. Results in Mathematics 71 (2017), 1167-1184.

11. K. Demirci, B. Kolay: A-Statistical Relative Modular Convergence of Positive Linear Operators. Positivity 21 (2017), 847-863.

12. F. DiRIK, P. OKÇU ŞAhIN: Statistical Relatively Equal Convergence and KorovkinType Approximation Theorem. Results in Mathematics 72 (2017), 1613-1621.

13. U. KADAK: Weighted Statistical Relative Invariant Mean in Modular Function Spaces with Related approximation Results. Numerical Functional Analysis and Optimization 39 (2018), 1181-1207.

14. S. Karakuş, K. Demirci, O. Duman: Statistical approximation by positive linear operators on modular spaces. Positivity 14 (2010), 321-334.

15. P.P. Konovkin: Linear Operators and Approximation Theory. Hindustan Publ. Co., Delhi, 1960.

16. W. M. KozLowski: Modular function spaces. Pure Appl. Math., Vol. 122, Marcel Dekker, Inc., New York, 1988.

17. K.. Kuratowski: Topology, Volls I and II. Academic Press, New York-London, 1966/1968.

18. I. MANTELLINI: Generalized sampling operators in modular spaces. Commentationes Math. 38 (1998), 77-92.

19. J. Musielak: Orlicz spaces and modular spaces. Lecture Notes in Mathematics, Vol. 1034 Springer-Verlag, Berlin, 1983.

20. J. MusielaK: Nonlinear approximation in some modular function spaces I. Math. Japon. 38 (1993), 83-90.

21. P. OKÇU ŞAhin, F. DIRIK: Statistical relative uniform convergence of double sequences of positive linear operators. Applied Mathematics E-Notes 17 (2017), 207-220.

22. S. ORHAN, K. Demirci: Statistical approximation by double sequences of positive linear operators on modular spaces. Positivity 19 (2015), 23-36.

23. B. Yilmaz, K. Demirci, S. Orhan: Relative Modular Convergence of Positive Linear Operators. Positivity 20 (2016), 565-577.

Sevda Yıldız

Faculty of Science and Arts 
Department of Mathematics

Sinop University

57000 Sinop, Turkey

sevdaorhan@sinop.edu.tr

Kamil Demirci

Faculty of Science and Arts

Department of Mathematics

Sinop University

57000 Sinop, Turkey

kamild@sinop.edu.tr 